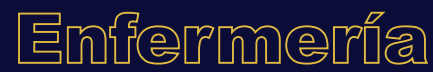

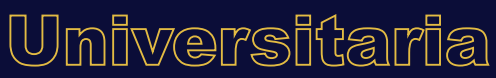

\section{Uso de sulfato de magnesio relacionado con la reanimación neonatal en prematuros}

\author{
Use of magnesium sulfate in the \\ neonatal reanimation of prematures
}

\section{Uso de sulfato de magnésio relacionado à ressuscitação neonatal em prematuros}

\section{P. Pino-Cortés ${ }^{a 1}$, D. Muena-Canales ${ }^{\text {b2 }}$, P. Gálvez-Ortega ${ }^{c_{3}}$}

ORCID

a $\underline{0000-0001-6714-5202}$

b $0000-0001-6550-4101$

c0000-0002-4847-5692

${ }^{1}$ Hospital San Juan de Dios, Santiago de Chile, Chile

${ }^{2}$ Hospital Clínico San Borja Arriarán, Santiago de Chile, Chile

3 Universidad de Chile, Facultad de Medicina, Departamento de Promoción de la Salud de la Mujer y del Recién Nacido, Santiago de Chile, Chile

Recibido: 15 abril 2020

Aceptado: 04 agosto 2020

\section{RESUMEN}

Introducción: La neuroprotección farmacológica se utiliza en el período antenatal para ayudar a disminuir el riesgo de parálisis cerebral o disfunciones motoras en el recién nacido (RN). El sulfato de magnesio $\left(\mathrm{MgSO}_{4}\right)$ es de gran utilidad por los beneficios que proporciona como tocolítico, neuroprotector fetal en prematuros menores a 32 semanas y en el manejo de la preeclampsia. Sin embargo, durante su uso se observaron efectos adversos a dosis dependientes tanto para la madre como para el RN, lo que genera gran importancia para el trabajo del profesional de obstetricia y enfermería.

* Autor para correspondencia. Correo electrónico: pagalvezo@uchile.cl https://doi.org/10.22201/eneo.23958421e.2021.2.869

1665-7063/@ 2021 Universidad Nacional Autónoma de México, Escuela Nacional de Enfermería y Obstetricia. Este es un artículo Open Access bajo la licencia CC BY-NC-ND (http://creativecommons.org/licenses/by-nc-nd/4.o/). 
Objetivo: Determinar la frecuencia de reanimación neonatal en prematuros menores de 32 semanas según el uso de neuroprotección con $\mathrm{MgSO}_{4}$.

Métodos: Estudio retrospectivo, observacional, realizado en un hospital de Santiago de Chile. Se realizó un muestreo aleatorio simple. Se incluyeron prematuros menores a 32 semanas de edad gestacional con o sin tratamiento de $\mathrm{MgSO}_{4}$ que hayan requerido o no reanimación neonatal. La información fue recogida a partir de fichas clínicas con un instrumento propio. Análisis de los datos por determinación de frecuencias absolutas y relativas de las variables y su comparación directa.

Resultados: Del total de los $\mathrm{RN}$ que recibieron $\mathrm{MgSO}_{4}, 61.7$ \% requirió reanimación neonatal al nacer, de los que no recibieron este neuroprotector, el 52.8 \% necesitó reanimación neonatal.

Conclusiones: La mayor frecuencia de reanimación neonatal está asociada al uso de la neuroprotección con $\mathrm{MgSO}_{4}$ y ocurre más probablemente en recién nacidos de edad gestacional menor que 32 semanas. Los resultados contribuyen a la toma de decisiones basadas en pruebas.

Palabras clave: Neuroprotección; parálisis cerebral; recién nacido prematuro; sulfato de magnesio; reanimación neonatal; Chile.

\section{ABSTRACT}

Introduction: Diverse drug-related neuroprotections are used during the prenatal period to help reduce the risk of cerebral palsy or motor dysfunctions in the newborn. Magnesium sulfate $\left(\mathrm{MgSO}_{4}\right)$ is useful as a fetal neuroprotector in prematures with less than 32 weeks, as well as in the management of preeclampsia. Nevertheless, some dose-dependent adverse effects both on the mother and the newborn have been reported. This situation is necessarily of concern to the nursing and obstetrics professional.

Objective: To determine the frequency of neonatal reanimation en prematures with less than 32 weeks with $\mathrm{MgSO}_{4}$ neuroprotection.

Methods: This is a retrospective and observational study conducted in a hospital in Santiago, Chile. Simple random sampling was used. Prematures with less than 32 weeks of gestational age, with or without $\mathrm{MgSO}_{4}$ treatment and who had or had not required neonatal reanimation, were included in the study. Data were collected from clinical records. Absolute and relative frequencies and their direct comparisons were calculated.

Results: From the total of newborns who received $\mathrm{MgSO}_{4}, 61.7 \%$ required neonatal reanimation, while from those who did not receive $\mathrm{MgSO}_{4}, 52.8 \%$ required neonatal reanimation.

Conclusions: The higher frequency of neonatal reanimation is associated with the received $\mathrm{MgSO}_{4}$ as a neuroprotection with and occurs more likely in neonates with a gestational age less than 32 weeks. The results contribute to evidence-based decision making.

Keywords: Neuroprotection; cerebral palsy; infant, premature; magnesium sulfate; neonatal reanimation; Chile.

RESUMO

Introdução: A neuroproteção farmacológica é utilizada no período pré-natal para ajudar a reduzir o risco de paralisia cerebral ou disfunções motoras no recém-nascido (RN). $\mathrm{O}$ sulfato de magnésio $\left(\mathrm{MgSO}_{4}\right)$ é muito útil devido aos benefícios que proporciona como 
tocolítico, neuroprotetor fetal em prematuros com menos de 32 semanas e no tratamento da pré-eclâmpsia. Porém, durante seu uso, foram registrados efeitos adversos em doses dependentes tanto para a mãe quanto para o recém-nascido, o que gera grande importância para o trabalho do profissional obstétrico e de enfermagem.

Objetivo: Determinar a frequência de reanimação neonatal em prematuros com menos de 32 semanas de acordo com o uso de neuroproteção com $\mathrm{MgSO}_{4}$.

Métodos: Estudo retrospectivo e observacional realizado em um hospital de Santiago de Chile. Foi realizada uma amostragem aleatória simples. Foram incluídos bebês prematuros com menos de 32 semanas de idade gestacional com ou sem tratamento com $\mathrm{MgSO}_{4}$ que exigia ou não a reanimação neonatal. Coletou-se informação de prontuários clínicos com um instrumento proprietário. Análise dos dados através da determinação das frequências absolutas e relativas das variáveis e sua comparação direta.

Resultados: Do total dos RN que receberam $\mathrm{MgSO}_{4}, 61,7 \%$ necessitaram de reanimação neonatal ao nascimento, dos que não receberam esse neuroprotetor, 52,8\% necessitaram de reanimação neonatal.

Conclusões: A frequência de reanimação neonatal foi maior em recém-nascidos com menos de 32 semanas de idade gestacional que receberam neuroproteção com $\mathrm{MgSO}_{4}$, situação semelhante em cada estrato de idade gestacional.

Palabras chave: Neuroproteção; paralisia cerebral; recém-nascido prematuro; sulfato de magnésio; reanimación neonatal; Chile.

\section{INTRODUCCIÓN}

La neuroprotección es el empleo de cualquier estrategia con el fin de prevenir una lesión neuronal', su uso en el periodo antenatal ayuda a disminuir el riesgo de parálisis cerebral (PC) o disfunciones motoras en el Recién Nacido (RN) $)^{2-6}$. Según la Guía Chilena Perinatal 2015, cuando se presentan amenazas de parto prematuro una de las estrategias neuroprotectoras es la administración de tocolíticos, corticoides y sulfato de magnesio, con cierta restricción para su aplicación, ya que no deben sumistrarse antes de las 24 ni después de las 34 semanas de gestación?. Por otro lado, la PC en países industrializados tiene una prevalencia de 2 a 2.5 / 1000 RN vivos. El antecedente de parto prematuro está presente en $35 \%$ de los neonatos con PC y el riesgo es 30 veces mayor en el RN pretérmino de muy bajo peso de nacimiento (menor a 1500 gramos) que en un RN de término que pesa más de 2500 gramos. Por consiguiente, la prevalencia de PC en los distintos países es influenciada por las tasas de prematuridad que estos poseen ${ }^{8-10}$.

La Organización Mundial de la Salud (OMS) estima que cada año nacen alrededor de 15 millones de prematuros a nivel mundial ${ }^{11}$. En Chile, de acuerdo con el Departamento de Estadísticas e Información de Salud (DEIS), en el año 2016, del total de nacidos vivos, 8.3 \% fueron RN menores a 37 semanas y $15.4 \%$ fueron menores a 32 semanas $^{12}$. Una investigación realizada en un hospital de Santiago de Chile señala que los principales factores asociados a nacimientos entre las 22 y 34 semanas son: la infección bacteriana ascendente en la clasificación de parto pretérmino espontáneo y la preeclampsia en la categoría interrupción del embarazo por indicación médica ${ }^{13,14}$. Según la prevalencia mencionada anteriormente, existe un importante porcentaje $(8.3 \%)^{12}$ que figura como población de riesgo para $\mathrm{PC}^{15}$, la cual requeriría administración de neuroprotector. 
El medicamento más usado en Chile para la neuroprotección antenatal es el sulfato de magnesio $\left(\mathrm{MgSO}_{4}\right)^{16-18}$, el cual ha sido utilizado para tratar y prevenir la eclampsia, así como por sus efectos tocolíticos en trabajos de parto pretérmino ${ }^{4,5,19,20}$. Aunado a su funcionalidad, se le atribuyó la neuroprotección en el feto y RN, pues a nivel cerebral evita los daños neuronales por hiperexcitabilidad, impidiendo la apoptosis en eventos de hipoxia cerebral, produce estabilización de la presión arterial, desarrollo del cerebro a través del factor de crecimiento nervioso, vasodilatación, efectos antiinflamatorio ${ }^{21}$ y además posee propiedades antioxidantes ${ }^{21-24}$. Según la Guía Chilena Perinatal 2015 y la Guía Clínica Prevención Parto Prematuro 2010, la administración de sulfato de magnesio está establecida vía intravenosa, con una dosis de carga de $4 \mathrm{gr} / \mathrm{h}$ y dosis de mantención de 1-2 gr/h, aplicada al menos 24 horas antes del parto, es importante suministrarlo entre las 24 a 34 semanas de gestación $n^{7,16}$.

Si bien el sulfato de magnesio ha sido de gran utilidad por los beneficios que aporta, se han registrado efectos adversos tanto para la madre como para el recién nacido. El ion magnesio puede ser tóxico para la madre en altas concentraciones ${ }^{25}$, en el feto existe la posibilidad que se presente dosis dependiente pues atraviesa la barrera placentaria de forma pasiva o facilitada ${ }^{22,26}$. Entre otros efectos que puede provocar en el feto están alteraciones tras su nacimiento a través de un bloqueo neuromuscular, con manifestaciones como alteraciones del patrón de deposiciones ${ }^{22}$, hipotonía, hiperreflexia y depresión respiratoria, lo que conlleva a la necesidad de reanimación al nacimiento, con un mayor uso de intubación endotraqueal y una alta probabilidad de ingreso a la unidad de neonatología $25,27,28$

A nivel mundial, existen escasas investigaciones que relacionan ambas variables (neuroprotección y reanimación neonatal). Los estudios realizados a la fecha principalmente son cuantitativos, los cuales incluyen diseños de tipo cohorte y ensayo clínico aleatorizado. El criterio de inclusión establecido en todos los estudios es una edad gestacional menor a 32 semanas. Entre las variables utilizadas, las principales fueron la necesidad de reanimación, la cual se describe según su complejidad (uso de ventilación, intubación, compresiones torácicas y aplicación de epinefrina) y el uso de sulfato de magnesio como neuroprotector. Los resultados de las publicaciones fueron similares, concluyen que no hay un aumento significativo en la necesidad de reanimación neonatal de los prematuros menores a 32 semanas que han recibido neuroprotección ${ }^{422,29}$.

Tras una revisión en las principales bases de búsqueda científica (Medline a través de PubMed, ScienceDirect, SciELO, LILACS) se aprecia que actualmente en Latinoamérica no existen investigaciones que involucren dichas variables (neuroprotección y reanimación neonatal). Generar evidencia respecto a este tema es de suma importancia, ya que la prevalencia de los prematuros en Chile ha ido incrementando en los últimos años. Según el Departamento de Estadísticas Nacional, el porcentaje de recién nacidos menores a 37 semanas ha variado del 2010 al 2016 de $7.2 \%$ a $8.3 \%$ 12,30,31. En consecuencia, se visualiza un aumento de los RN que requieren neuroprotección, haciendo cada vez más necesario conocer los posibles efectos que los medicamentos neuroprotectores tienen de acuerdo con las dosis utilizadas en el país ${ }^{32}$. La relevancia de este estudio se enfoca en la reducción de la morbilidad y mortalidad neonatal ${ }^{33}$, además de mejorar la calidad de vida del RN a través de los resultados entregados. Asimismo, invita a la realización de estudios analíticos con asociación de estas variables para reafirmar a nivel local la evidencia aportada por los estudios extranjeros mencionados anteriormente, ya que sus resultados no se pueden extrapolar a nivel global por los distintos contextos y normas de las poblaciones estudiadas ${ }^{34}$. Este estudio se realiza con el fin de 
aportar datos latinoamericanos respecto a la temática en estudio, de la cual existe casi nula evidencia para el contexto latino.

El objetivo de esta investigación es determinar la frecuencia de reanimación neonatal y el uso de neuroprotección con sulfato de magnesio en prematuros menores a 32 semanas de la Unidad de Neonatología de un hospital de Santiago de Chile, entre los años 2014 y 2019.

\section{MÉTODOS}

Estudio de carácter observacional descriptivo; se basa en una revisión y recaudación de datos provenientes de fichas clínicas de recién nacidos admitidos entre los años 2014-2019 en la Unidad de Neonatología de un hospital de Santiago de Chile. Este hospital se caracteriza por ser de alta complejidad y recibe a pacientes pertenecientes a 5 comunas de Santiago de Chile, además de las derivaciones de otros siete hospitales de la Red de Salud que son de mediana y menor complejidad.

En el estudio se incluyeron prematuros menores a 32 semanas de edad gestacional. El universo estuvo compuesto por 453 neonatos y se realizó un muestreo simple analizando a 209 de ellos. De estos se excluyeron recién nacidos con anomalía congénita cardiaca o pulmonar, con antecedente prenatal de restricción de crecimiento intrauterino, embarazo gemelar, uso de sulfato de magnesio en dosis no equivalentes a las usadas en neuroprotección, desprendimiento prematuro de placenta normoinserta (DPPNI), consumo de drogas o alcohol durante el embarazo y patologías maternas que incluyen diabetes pregestacional e hipertensión arterial crónica. Las variables principales de esta investigación son la administración del sulfato de magnesio como neuroprotector en el periodo antenatal y la necesidad de emplear maniobras de reanimación en el recién nacido.

Por otro lado, se analizaron antecedentes maternos como: edad, estado civil, patologías concomitantes del embarazo (síndrome hipertensivo del embarazo, diabetes gestacional e infecciones maternas). Asimismo, antecedentes prenatales y del parto como: rotura prematura de membranas (RPM), administración de corticoides para maduración pulmonar, vía del parto, antecedentes de la atención inmediata del recién nacido como edad gestacional según examen físico, sexo, APGAR (al minuto y a los cinco minutos), reanimación neonatal (tipo de ventilación a presión positiva, compresiones torácicas o drogas) y defunción.

Del total de 209 registros de recién nacidos menores a 32 semanas de edad gestacional, 107 fueron incluidos en el estudio y 102 quedaron fuera de este según criterios de exclusión, principalmente por tratarse de un embarazo gemelar, desprendimiento de placenta normo inserta, restricción de crecimiento intrauterino y madre hipertensa crónica. De las 107 fichas clínicas que cumplieron con el criterio de selección, 7 se encontraban sin la ficha neonatal por lo que no fue posible rescatar sus datos, quedando un total de 100 (Figura 1). Cabe mencionar que algunos datos no fueron registrados en un porcentaje mínimo ( $2 \%$ ) de fichas clínicas, por ende, no se indican como resultados.

Para la recolección de datos, se elaboró un instrumento que consta de tres dimensiones, las cuales son: a. Variables sociodemográficas maternas, b. Antecedentes prenatales y del parto, c. Antecedentes de la atención inmediata del recién nacido. Estas dimensiones contienen apartados, los cuales desglosan cada temática para facilitar la recolección de antecedentes necesarios y así cumplir con los objetivos de la investigación. El instrumento fue aplicado por los investigadores del estudio. Este tipo de herramienta no requiere validación por expertos, ya que sólo pretende recolectar la información extraída desde la fuente secundaria (ficha clínica). Para el análisis estadístico se utilizó 
No cumplen con criterio de selección $=102$
Universo de pacientes evaluados para

elegibilidad

$\mathrm{N}=453$

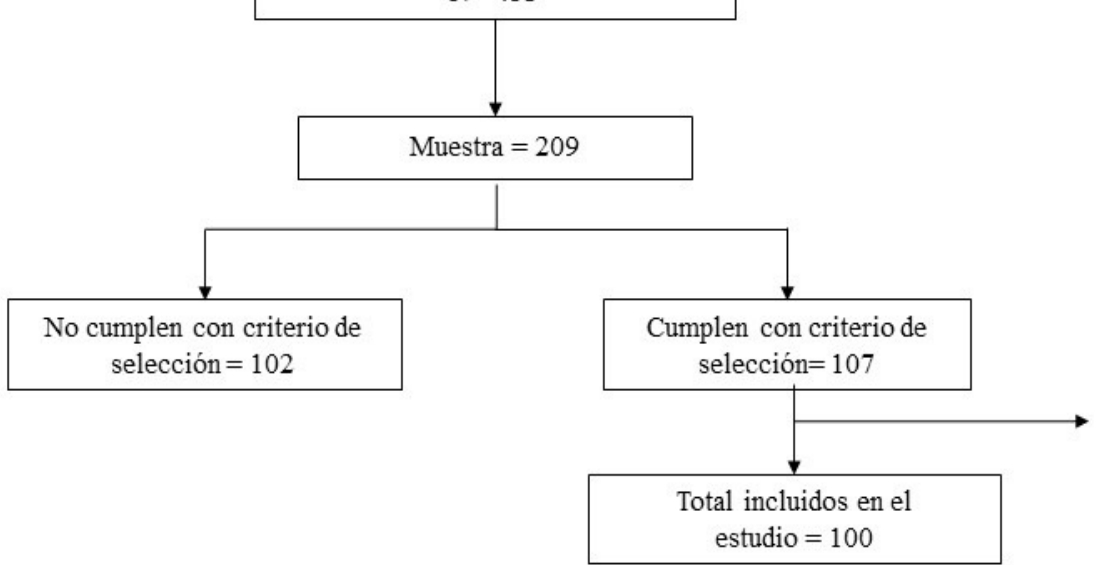

Ficha clínica sin datos de periodo neonatal $=7$

Figura 1. Diagrama de flujo

Stata 13.0. Se determinó frecuencia absoluta y relativa en variables tanto cuantitativas como cualitativas. Se determinó medidas de tendencia central y dispersión en variables cuantitativas continuas.

Dentro de las consideraciones éticas, con fecha 22 de enero del año 2019, se autorizó la eximición del uso de un consentimiento informado para acceder a las fichas clínicas emitido por el Comité de Ética de Investigaciones en Seres Humanos de la Facultad de Medicina de la Universidad de Chile y la Unidad de Investigación del hospital en cuestión, se resguardó el anonimato y el buen uso de la información en beneficio de la sociedad.

\section{RESULTADOS}

Respecto a las características sociodemográficas, la edad mínima materna fue de 14 años, la máxima de 45 años con una media de 28 años, $\mathrm{DE}= \pm 6$ años. El estado civil se encontraba especificado sólo en $36 \%$ del total de fichas clínicas, de este porcentaje, $30.6 \%$ de las mujeres se encontraban solteras, $16.7 \%$ casadas y $52.8 \%$ en unión libre. Los antecedentes prenatales y del parto se exponen en la tabla 1.

De las infecciones maternas, 50 \% correspondió a corioamnionitis, $45.8 \%$ infecciones del tracto urinario y $4.2 \%$ fueron otras infecciones no especificadas. $65.2 \%$ recibió tratamiento con antibiótico, el cual no se explicitaba en los registros de antecedentes prenatales.

La administración de corticoide antenatal se aplicó en $85 \%$ del total de quienes recibieron dosis terapéutica para la maduración pulmonar. En la dosificación al $56.5 \%$ se le ministró doble dosis, $29.4 \%$ unidosis, $12.9 \%$ tres dosis y $1.2 \%$ cuatro dosis. $\mathrm{El} \mathrm{MgSO}_{4}$ para la neuroprotección fue administrado en $47 \%$ del total de las gestantes, con una media de 3.9 horas y $\mathrm{DE}= \pm 3.8$ horas.

Las variables implicadas en la atención inmediata del recién nacido se encuentran en las tablas 2 y 3 . La edad gestacional mínima fue de 24 semanas y la máxima de 31 semanas. De acuerdo con el puntaje APGAR obtenido al minuto de vida de los RN que recibieron neuroprotección, 12.8 \% estuvo 


\section{Tabla 1. Antecedentes prenatales y del parto}

\begin{tabular}{lc} 
Antecedentes prenatales & Porcentaje ( \% ) \\
\hline SHE & 21.0 \\
\hline DG & 11.0 \\
\hline Infección materna & 24.0 \\
\hline Corticoides & 85.0 \\
\hline RPM & 38.0 \\
\hline$<18$ horas & 39.5 \\
\hline$>18$ horas & 57.9 \\
\hline Administración de MgSO 4 & 47.0 \\
\hline Carga & 34.0 \\
\hline Carga + mantención & 61.7 \\
\hline Antecedentes del parto & \\
\hline Parto vaginal & 48.0 \\
\hline Cesárea & 52.0
\end{tabular}

entre 0-3 puntos, poco más de una cuarta parte entre 4-6 puntos y $59.6 \%$ entre 7-10 puntos. A los 5 minutos de vida, el puntaje entre 0-3 puntos fue de $0 \%$, de 4-6 puntos fue de $8.5 \%$ y entre 7-10 puntos fue de $91.5 \%$. Por otra parte, de los neonatos sin administración antenatal de $\mathrm{MgSO}_{4}$, el puntaje APGAR logrado al minuto de vida fue de $22.6 \%$ entre 0-3 puntos, $18.9 \%$ entre $4-6$ puntos y $56.6 \%$ entre 7-10 puntos. A los 5 minutos de vida, o \% entre 0-3 puntos, $11.3 \%$ entre $4-6$ puntos y $86.8 \%$ entre 7-10 puntos (existió 1.9 \% de RN sin neuroprotección razón por la cual se excluyeron los datos de puntaje APGAR en sus antecedentes).

Tabla 2. Atención inmediata del recién nacido

\begin{tabular}{lrl} 
Variable del recién nacido & Porcentaje $(\%)$ & Media \pm DE \\
Peso (gramos) $n=98$ & & \\
$\geq 2500$ & 31.0 & $2767.5 \pm 95.5$ \\
\hline$<2500$ & 56.0 & $1706.2 \pm 148.5$ \\
\hline$<1500$ & 11.0 & $288.6 \pm 137.3$ \\
\hline$<1000$ & & $846.3 \pm 124.4$ \\
\hline Edad gestacional (semanas) & & \\
$32-31$ & 57.0 & \\
\hline $30-29$ & 25.0 & \\
\hline$<28$ & 18.0 & \\
\hline Sexo $n=99$ & & \\
\hline Femenino & 37.0 & \\
\hline Masculino & 63.0 & \\
\hline
\end{tabular}




\begin{tabular}{lc}
\hline Reanimación neonatal & \\
Reanimados & 57.0 \\
\hline VNI & 47.4 \\
\hline VNNI & 50.9 \\
\hline CT & 12.3 \\
\hline Drogas & 1.7 \\
\hline Muerte & 0 \\
\hline
\end{tabular}

Nota. DE: Desviación Estándar; VNI: Ventilación Neonatal Invasiva; VNNI: Ventilación Neonatal No Invasiva; CT: Compresiones Torácicas

Tabla 3. Porcentaje de puntaje APGAR otorgado al minuto y a los 5 minutos de vida según edad gestacional

Edad gestacional (semanas)

\begin{tabular}{llll}
\hline \multicolumn{1}{c}{ APGAR } & $\begin{array}{c}32-31 \\
\mathrm{n}=57\end{array}$ & $\begin{array}{c}30-29 \\
\mathrm{n}=25\end{array}$ & $\begin{array}{c}<28 \\
\mathrm{n}=18\end{array}$ \\
\hline Al minuto de vida & $\%(\mathrm{n})$ & $\%(\mathrm{n})$ & $\%(\mathrm{n})$ \\
\hline $0-3$ & $15.8(9)$ & $16.0(4)$ & $33.3(6)$ \\
\hline $4-6$ & $15.8(9)$ & $24.0(6)$ & $44.5(8)$ \\
\hline $7-10$ & $68.4(39)$ & $60.0(15)$ & $22.2(4)$ \\
\hline A los 5 minutos de vida & & & $0(0)$ \\
\hline $0-3$ & $0(0)$ & $0(0)$ & $11.1(2)$ \\
\hline $4-6$ & $8.8(5)$ & $12.0(3)$ & $88.9(16)$ \\
\hline $7-10$ & $91.2(52)$ & $88.0(22)$ & \\
\hline
\end{tabular}

Con respecto a la frecuencia de neuroprotección y la necesidad de reanimación neonatal (Tabla 4), del total de los RN a los cuales se les administró sulfato de magnesio, 61.7 \% requirió reanimación neonatal al nacer, mientras que poco más de la mitad de los neonatos sin recibir la neuroprotección necesitó reanimación neonatal.

\begin{tabular}{|c|c|c|c|c|c|c|}
\hline \multirow{4}{*}{$\begin{array}{l}\text { Edad gestacional } \\
\quad \text { (semanas) }\end{array}$} & \multicolumn{6}{|c|}{$\begin{array}{l}\text { Tabla 4. Neuroprotección }\left(\mathrm{MgSO}_{4}\right) \text { y necesidad } \\
\text { de reanimación neonatal según edad gestacional }\end{array}$} \\
\hline & \multicolumn{3}{|c|}{ Con neuroprotección } & \multicolumn{3}{|c|}{ Sin neuroprotección } \\
\hline & \multicolumn{2}{|r|}{ Reanimados } & \multicolumn{2}{|l|}{$\begin{array}{l}\text { No } \\
\text { reanimados }\end{array}$} & Reanimados & $\begin{array}{l}\text { No } \\
\text { reanimados }\end{array}$ \\
\hline & $\mathbf{n}$ & & & $\mathbf{n}$ & & \\
\hline $32-31$ & 23 & $56.5(13)$ & $43.5(10)$ & 34 & $44.1(15)$ & $55.9(19)$ \\
\hline $30-29$ & 12 & $58.3(7)$ & $41.7(5)$ & 13 & $53.8(7)$ & $46.2(6)$ \\
\hline$<28$ & 12 & $75(9)$ & $25(3)$ & 6 & $100(6)$ & $0(0)$ \\
\hline Total & 47 & $61.7(29)$ & $38.3(18)$ & 53 & $52.8(28)$ & $47.2(25)$ \\
\hline
\end{tabular}

Nota. $\mathrm{MgSO}_{4}$ : Sulfato de magnesio 


\section{DISCUSIÓN}

De conformidad con las variables principales, el estudio muestra que $61.7 \%$ de los RN con neuroprotección requirió reanimación neonatal durante la atención inmediata, porcentaje que supera en 8.9 \% a los RN sin neuroprotección con necesidad de reanimación. Al compararlo con investigaciones similares que existen a nivel mundial, es relevante destacar que en un ensayo clínico aleatorizado en el cual se estudiaron 1047 neonatos, $70.3 \%$ de los RN con neuroprotección y $74.6 \%$ de los RN sin neuroprotección requirieron reanimación neonatal ${ }^{29}$. Asimismo, en otro estudio de cohorte en el que se incluyeron 107 neonatos, $60.7 \%$ de los RN con neuroprotección y $64.7 \%$ sin neuroprotección requirieron reanimación neonatal ${ }^{22}$; evidenciando en ambas investigaciones mayor frecuencia de reanimación en RN con ausencia de neuroprotección, datos opuestos en nuestra investigación.

Por otra parte, existe un estudio de cohorte en el cual se estudiaron 6015 neonatos, cuyo porcentaje de RN reanimados que recibieron neuroprotección fue mayor a los que no la recibieron (95.7 \% para aquellos expuestos a $\mathrm{MgSO}_{4}$ y $93.7 \%$ a quienes no se les aplicó sulfato de magnesio pero que ambos requirieron reanimación neonatal)4, datos similares a los obtenidos en el presente estudio. No obstante, a pesar de los diferentes valores presentados en los tres estudios mencionados, todos concluyen que no hubo un aumento significativo en la necesidad de reanimación neonatal ante el uso de sulfato de magnesio como neuroprotector. Estos resultados podrían indicar que no habría relación entre las variables mencionadas, ya que el fármaco no afectaría el resultado; sin embargo, para poder comprobar esta hipótesis es necesario realizar un estudio analítico.

Respecto a la prueba de APGAR, se observan diferencias en caso de administración o no del sulfato de magnesio; no obstante, se identifica que el medicamento no afecta dicha valoración. Esto solo puede ser verificado en un estudio analítico como lo indicado en una investigación que mide indirectamente esta variable y no encuentra relación estadísticamente significativa ${ }^{35}$.

Una de las principales fortalezas de esta investigación consiste en los criterios de exclusión establecidos que incorporan factores que pueden incrementar la necesidad de reanimación neonatal ${ }^{36}$, debido al posible daño al bienestar fetal ocasionado por la depresión respiratoria neonatal. En consecuencia, se descartaron a los neonatos con antecedentes perinatales, variables que pudieran influir y desviar los resultados. Es posible inferir que la frecuencia de reanimación neonatal se explica principalmente por la administración de $\mathrm{MgSO}_{4}$. En los resultados que se obtuvieron, la frecuencia de reanimados es mayor en mujeres con administración de neuroprotección fetal, por ello se podría afirmar que esto se debe a la administración del fármaco. Sin embargo, es de suma importancia la realización de un estudio analítico para verificar si este resultado y la diferencia de porcentajes están relacionados estadísticamente.

\section{CONCLUSIÓN}

La frecuencia de reanimación neonatal fue mayor en los recién nacidos menores a 32 semanas de edad gestacional que recibieron neuroprotección con sulfato de magnesio (siendo superior en cada estratificación por edad gestacional presentada en los resultados), dando respuesta al objetivo principal de la investigación.

La relevancia de este artículo es aportar al conocimiento científico nacional y latinoamericano sobre el uso de $\mathrm{MgSO}_{4}$ y la reanimación neonatal, si bien existen investigaciones internacionales, estas no se pueden extrapolar a la realidad latinoamericana y chilena. Por otra parte, se justifica incorporar estos estudios en Chile debido al progresivo aumento en el número de nacimientos de RN prematuros, quienes pueden requerir aplicación de neuroprotección fetal según corresponda. Por 
consiguiente, es esencial determinar los posibles efectos de la administración de $\mathrm{MgSO}_{4} \mathrm{y}$ utilizarlo dentro de los márgenes de seguridad tanto para la madre como para el RN.

La relevancia de nuestros resultados radica en que el profesional de obstetricia y enfermería debe ser capaz de tener conocimientos actualizados para que la toma de decisiones sea segura para los neonatos y sus madres. En ese sentido, el conocer las ventajas y desventajas del uso de sulfato de magnesio en neonatos pretérmino reanimados permite a estos profesionales tomar decisiones basadas en la evidencia actualizada, considerando los riesgos asociados a la seguridad de las gestantes y sus hijos o hijas.

El presente estudio es el paso inicial para la apertura de nuevas líneas de investigación que puedan llevar a cabo un análisis y consiguiente relación de los posibles efectos del $\mathrm{MgSO}_{4}$ en el feto, sobre todo en su aplicación para la neuroprotección. Se sugiere replicar los estudios en diversas localidades del país, ya que cada una de estas presenta distintos contextos, a fin de lograr una visión más completa del país en su totalidad. Aunado a esto, se recomienda llevar previamente un registro cauteloso y detallado de la administración de $\mathrm{MgSO}_{4}$ como neuroprotector e incorporar una monitorización de los niveles plasmáticos maternos en pre partos de este fármaco para obtener un análisis respecto a los efectos adversos en el recién nacido que dependen de las dosis propiciadas antenatalmente.

Las limitaciones que existieron estuvieron relacionadas con la recaudación de fichas clínicas del recién nacido. Gran parte de estas se encontraban retenidas por seguimiento del prematuro en nivel secundario y también por problemas de logística en la unidad de archivos. Además, en la revisión de fichas clínicas fue limitante la obtención de datos provenientes de estas por ilegibilidad de la letra en el registro, la omisión de datos requeridos por el instrumento de recolección y la presencia de fichas sin registro del periodo neonatal, presentando sólo registro del seguimiento. El manejo de dichas fichas fue por medio de la omisión de los datos ilegibles o no registrados, sin desechar uso de los demás datos del recién nacido y en caso de ausencia de ficha neonatal, se descartaron en su totalidad. Es necesario destacar que existió una limitación que afectó directamente la aplicación del instrumento y los resultados del estudio, ya que no existe una regularización en el hospital sobre el registro en la ficha clínica perinatal del recién nacido respecto a la administración de sulfato de magnesio, el cual no establece detalle de: su utilización, las dosis administradas y las horas infusión.

\section{RESPONSABILIDADES ÉTICAS}

Protección de personas y animales. No se realizaron intervenciones en personas ni animales.

Confidencialidad. La información extraída de las fichas solo fue manejada por los investigadores y se codificaron las fichas para no correlacionar con nombres de las personas.

Conflicto de intereses. Los autores declaran no tener conflicto de intereses.

Financiamiento. Ninguno.

\section{REFERENCIAS}

1. Richter AE, Scherjon SA, Dikkers R, Bos AF, Kooi EMW. Antenatal magnesium sulfate and preeclampsia differentially affect neonatal cerebral oxygenation. Neonatology. 2020; 117(3): 331-40. https://doi.org/10.1159/000507705

2. Bachnas MA, Akbar MIA, Dachlan EG, Dekker G. The role of magnesium sulfate $\left(\mathrm{MgSO}_{4}\right)$ in fetal neuroprotection. J Matern Fetal Neonatal Med. 2019; 34(6): 966-78.

https://doi.org/10.1080/14767058.2019.1619688 
3. Magee LA, De Silva DA, Sawchuck D, Synnes A, Von Dadelszen P. No. 376-Magnesium sulphate for fetal neuroprotection. J Obstet Gynaecol Can. 2019; 41(4): 505-22.

https://doi.org/10.1016/j.jogc.2018.09.018

4. Weisz DE, Shivananda S, Asztalos E, Yee W, Synnes A, Lee SK, et al. Intrapartum magnesium sulfate and need for intensive delivery room resuscitation. Arch Dis Child Fetal Neonatal Ed. 2015; 100: F59-65. https://doi.org/10.1136/archdischild-2013-305884

5. Chollat C, Marret S. Magnesium sulfate and fetal neuroprotection: Overview of clinical evidence. Neural Regen Res. 2018; 13(12): 2044-9. https://doi.org/10.4103/1673-5374.241441

6. Oddie S, Tuffnell DJ, McGuire W. Antenatal magnesium sulfate: Neuro-protection for preterm infants. Arch Dis Child Fetal Neonatal Ed. 2015; 100(6): F553-7.

https://doi.org/10.1136/archdischild-2014-307655

7. Ministerio de Salud. Guía perinatal. Chile: MINSAL; 2015. https://bit.ly/3ohLyWE

8. Gómez-López S, Jaimes VH, Palencia-Gutiérrez CM, Hernández M, Guerrero A. Parálisis cerebral infantil. Arch Venez Puer Ped. 2013; 76(1): 30-9. https://bit.ly/3CM4UI6

9. Herman S, Santoso B, Joewono HT, Sulistyono A, Usman AN. Relationship between early preterm birth (22-33 weeks) and late preterm birth (34-36 weeks) with the characteristics of sociodemography in primiparous and multiparous. Enferm clín. 2020; 30(Suppl 2): 541-6.

https://doi.org/10.1016/j.enfcli.2019.07.156

10. Leijser LM, De Vries LS. Preterm brain injury: Germinal matrix-intraventricular hemorrhage and post-hemorrhagic ventricular dilatation. Handb Clin Neurol. 2019; 162: 173-99.

https://doi.org/10.1016/B978-0-444-64029-1.00008-4

11. Organización Mundial de la Salud. Nacimientos prematuros. Ginebra: OMS; 2018.

12. Departamento de Estadísticas e Información de Salud (DEIS). Nacimientos inscritos según edad de la madre por región y comuna de residencia de la madre. Santiago de Chile: DEIS; 2016.

13. Ovalle A, Kakarieka E, Díaz M, García-Huidobro T, Acuña MJ, Morong C, et al. Mortalidad perinatal en el parto prematuro entre 22 y 34 semanas en un hospital público de Santiago, Chile. Rev. chil. obstet. ginecol. 2012; 77(4): 263-70. http://dx.doi.org/10.4067/So717-75262012000400005

14. Ovalle A, Kakarieka E, Rencoret G, Fuentes A, Del Río MJ, Morong C, et al. Factores asociados con el parto prematuro entre 22 y 34 semanas en un hospital público de Santiago. Rev. méd. Chile. 2012; 140(1): 19-29. http://dx.doi.org/10.4067/So034-98872012000100003

15. Muñoz-Martínez C, Parra-Pineda MO. Sulfato de magnesio para neuroprotección fetal: revisión de la literatura. Rev Colomb Obstet Ginecol. 2014; 65(3): 215-27. http://dx.doi.org/10.18597/rcog.49

16. Ministerio de Salud. Guía clínica Prevención Parto Prematuro. Santiago de Chile: MINSAL; 2010. https://bit.ly/3lug94w

17. Chiarello DI, Marín R, Proverbio F, Coronado P, Toledo F, Salsoso R, et al. Mechanisms of the effect of magnesium salts in preeclampsia. Placenta. 2018; 69: 134-9.

https://doi.org/10.1016/j.placenta.2018.04.011

18. Lingam I, Robertson NJ. Magnesium as a neuroprotective agent: A review of its use in the fetus, term infant with neonatal encephalopathy, and the adult stroke patient. Dev Neurosci. 2018; 4O(1): 1-12. https://doi.org/10.1159/000484891

19. Chollat C, Sentilhes L, Marret S. Fetal neuroprotection by magnesium sulfate: From translational research to clinical application. 2018; 9: 1-7. https://doi.org/10.3389/fneur.2018.00247 
20. Freret TS, James KE, Little SE, Kaimal AJ. 365: Does magnesium prophylaxis affect maternal and neonatal outcomes among women with preeclampsia without severe features? Am J Obstet Gynecol. 2020; 222(1): S243. https://doi.org/10.1016/j.ajog.2019.11.381

21. Daher I, Le Dieu-Lugon B, Dourmap N, Lecuyer M, Ramet L, Gomila C, et al. Magnesium sulfate prevents neurochemical and long-term behavioral consequences of neonatal excitotoxic lesions: Comparison between male and female mice. J Neuropathol Exp Neurol. 2017; 76(10): 883-97.

https://doi.org/10.1093/jnen/nlxo73

22. Lloreda-Garcia JM, Lorente-Nicolás A, Bermejo-Costa F, Martínez-Uriarte J, López-Pérez R. Necesidad de reanimación en prematuros menores de 32 semanas expuestos a sulfato de magnesio para neuroprotección fetal. Rev. chil. pediatr. 2016; 87(4): 261-7.

http://dx.doi.org/10.1016/j.rchipe.2015.11.006

23. Itoh K, Maki T, Shindo A, Egawa N, Liang AC, Itoh N, et al. Magnesium sulfate protects oligodendrocyte lineage cells in a rat cell-culture model of hypoxic-ischemic injury. Neurosci Res. 2016; 106: 66-9. https://doi.org/10.1016/j.neures.2015.12.004

24. Ozen M, Xie H, Shin N, Al-Yousif G, Clemens J, McLane MW, et al. Magnesium sulfate inhibits inflammation through $\mathrm{P}_{2} \mathrm{X} 7$ receptors in human umbilical vein endothelial cells. Pediatr Res. 2020; 87(3): 463-71. https://doi.org/10.1038/s41390-019-0557-7

25. Abbassi-Ghanavati M, Alexander JM, McIntire DD, Savani RC, Leveno KJ. Neonatal effects of magnesium sulfate given to the mother. Am J Perinatol. 2012; 29(10): 795-800.

https://doi.org/10.1055/s-0032-1316440

26. Kawasaki K, Kondoh E, Chigusa Y, Kawamura Y, Mogami H, Takeda S, et al. Metabolomic profiles of placenta in preeclampsia. Antioxidant effect of magnesium sulfate on trophoblasts in earlyonset preeclampsia. Hypertension. 2019; 73(3): 671-9.

https://doi.org/10.1161/HYPERTENSIONAHA.118.12389

27. De Jesus LC, Sood BG, Shankaran S, Kendrick D, Das A, Bell EF, et al. Antenatal magnesium sulfate exposure and acute cardiorespiratory events in preterm infants. Am J Obstet Gynecol. 2015; 212(1): 1-13. https://doi.org/10.1016/j.ajog.2014.07.023

28. Maged AM, Elsherief A, Hassan H, Salaheldin D, Omran KA, Almohamady M, et al. Maternal, fetal, and neonatal outcomes among different types of hypertensive disorders associating pregnancy needing intensive care management. J Matern Fetal Neonatal Med. 2020; 33(2): 314-21.

https://doi.org/10.1080/14767058.2018.1491030

29. Drassinower D, Friedman AM, Levin H, Običan SG, Gyamfi-Bannerman C. Does magnesium exposure affect neonatal resuscitation? Am J Obstet Gynecol. 2015; 213(3): 1-19.

https://doi.org/10.1016/j.ajog.2015.05.052

30. Departamento de Estadísticas e Información de Salud. Natalidad y Mortalidad 2014. Santiago de Chile: DEIS; 2016.

31. Araya BM, Díaz M, Paredes D, Ortiz J. Association between preterm birth and its subtypes and maternal sociodemographic characteristics during the post-transitional phase in a developing country with a very high human development index. Public Health. 2017; 147: 39-46. https://doi.org/10.1016/j.puhe.2017.01.027

32. Solevåg AL, Schmölzer GM, Y-Cheung P. Novel interventions to reduce oxidative-stress related brain injury in neonatal asphyxia. Free Radic Biol Med. 2019; 142: 113-22.

https://doi.org/10.1016/j.freeradbiomed.2019.04.028 
33. Nuss EE, Spiegelman J, Turitz AL, Gyamfi-Bannerman C. Childhood neurodevelopment after spontaneous versus indicated preterm birth. Am J Obstet Gynecol MFM. 2020; 2(2): 1-7.

https://doi.org/10.1016/j.ajogmf.2019.100082

34. Wolfe HA, Morgan RW, Zhang B, Topjian AA, Fink EL, Berg RA, et al. Deviations from AHA guidelines during pediatric cardiopulmonary resuscitation are associated with decreased event survival. Resuscitation. 2020; 149: 89-99. https://doi.org/10.1016/j.resuscitation.2020.01.035

35. Kim SH, Kim YJ, Shin SH, Cho H, Shin SH, Kim EK, et al. Antenatal magnesium sulfate and intestinal morbidities in preterm infants with extremely low gestational age. Pediatr Neonatol. 2021; 62(2): 202-7. https://doi.org/10.1016/j.pedneo.2020.12.009

36. Sawyer T, Lee HC, Aziz K. Anticipation and preparation for every delivery room resuscitation. Semin Fetal Neonatal Med. 2018; 23(5): 312-20. https://doi.org/10.1016/j.siny.2018.06.004 